\title{
THE FIRST VARIATION OF A FUNCTIONAL*
}

\author{
BY W. G. SMILEY, JR., AND G. C. EVANS
}

1. Introduction. The purpose of the present note is to present simple hypotheses which are sufficient to yield the two fundamental forms for the variation of a functional, expressed by a Stieltjes and a Lebesgue integral respectively. $\dagger$ The functional $F\left[f\left(\begin{array}{c}b \\ a\end{array}\right)\right]$ is supposed to be defined for continuous functions $f(x)$ within a region $R$ bounded by the continuous functions $\Phi_{1}(x)$, $\Phi_{2}(x)$, where $\Phi_{1}(x)<\Phi_{2}(x)$, and by the ordinates $x=a, x=b$ :

$$
\Phi_{1}(x)<f(x)<\Phi_{2}(x), \quad a \leqq x \leqq b .
$$

The following hypotheses are to be considered:

(I) There is an $M_{1}$ such that

$$
\left|F\left[f_{1}\right]-F\left[f_{2}\right]\right| \leqq M_{1} \max \left|f_{1}-f_{2}\right|, \quad\left(f_{1}, f_{2} \text { in } R\right) .
$$

(II) The first variation

$$
D\left[f_{1}, \phi\right]=\lim _{\epsilon=0} \frac{F\left[f_{1}+\epsilon \phi\right]-F\left[f_{1}\right]}{\epsilon}
$$

exists, and the limit so defined exists uniformly for all $f_{1}(x)$ in $R$, where $\phi(x)$ is an arbitrary given continuous function, $a \leqq x \leqq b$.

(III) There is an $M$ such that

* Presented to the Society, February 22, 1930.

$\dagger$ The reader may consult the following references for various types of sufficient conditions:

V. Volterra, Les Fonctions de Lignes, Paris, 1911.

F. Riesz, Concernant les opérations fonctionnelles linéaires, Annales de l'Ecole Normale Supérieure, vol. 31, p. 10. See also F. Riesz, Sur les opérations lineaires (troisième note), Annales de l'Ecole Normale Supérieure, vol. 8 (1907), p. 439.

M. Fréchet, Sur la notion de différentielle de fonction de ligne, Transactions of this Society, vol. 15 (1914), p. 139.

G. C. Evans, Note on the derivative and the variation of a function depending on all the values of another function, this Bulletin, vol. 21 (1915), p. 387.

P. J. Daniell, The derivative of a functional, this Bulletin, vol. 25 (1919), p. 414 . 


$$
\left|F\left[f_{1}\right]-F\left[f_{2}\right]\right| \leqq M \int_{a}^{b}\left|f_{1}(x)-f_{2}(x)\right| d x, \quad\left(f_{1}, f_{2} \text { in } R\right) .
$$

The hypothesis (III) evidently implies (I), with $M_{1}=M(b-a)$.

THEOREM 1. With the hypotheses (I), (II) the first variation takes the form

$$
D[f, \phi]=\int_{a}^{b} \phi(x) d \alpha_{f}(x)
$$

where $\alpha_{f}(x)$ is a function of limited variation of $x$ which for a given $f$ is independent of $\phi$.

THEOREM 2. With the hypotheses (II), (III) the first variation takes the form

$$
D[f, \phi]=\int_{a}^{b} \phi(x) \beta_{f}(x) d x,
$$

where $\beta_{f}(x)$ is summable in the Lebesgue sense, and for a given $f$ is independent of $\phi$.

2. Proof of Theorem 1. In order to prove Theorem 1 it is sufficient to prove that $D[f, \phi]$ is additive for $\phi$; for then

$$
D\left[f, c_{1} \phi_{1}+c_{2} \phi_{2}\right]=c_{1} D\left[f, \phi_{1}\right]+c_{2} D\left[f, \phi_{2}\right],
$$

for arbitrary constants $c_{1}, c_{2}$. That the Stieltjes integral form of representation follows from this fact and the inequality $|D[f, \phi]| \leqq M_{1} \max |\phi|$, which is an immediate consequence of (I), is Riesz's well known theorem.

From the inequalities which express (II):

$$
\begin{aligned}
& \left|D\left[f_{1}, \phi\right]-\frac{F\left[f_{1}+\epsilon \phi\right]-F\left[f_{1}\right]}{\epsilon}\right|<\eta(\epsilon), \\
& \left|D\left[f_{2}, \phi\right]-\frac{F\left[f_{2}+\epsilon \phi\right]-F\left[f_{2}\right]}{\epsilon}\right|<\eta(\epsilon),
\end{aligned}
$$

in which the infinitesimal $\eta(\epsilon)=\eta_{\phi}(\epsilon)$ depends on $\phi$ but not on $f_{i}$, it follows that

$$
\left|D\left[f_{1}, \phi\right]-D\left[f_{2}, \phi\right]\right|<2 \eta(\epsilon)+2 M \frac{\max \left|f_{1}-f_{2}\right|}{\epsilon} .
$$


By first limiting $\epsilon$ and then $\left|f_{1}-f_{2}\right|$, we see that this inequality implies that for a given $\phi, D[f, \phi]$ is uniformly continuous in $f$.

But in the equation

$$
\text { (3) } \begin{aligned}
& \frac{F\left[f+\epsilon\left(\phi_{1}+\phi_{2}\right)\right]-F[f]}{\epsilon}= \frac{F\left[f+\epsilon\left(\phi_{1}+\phi_{2}\right)\right]-F\left[f+\epsilon \phi_{1}\right]}{\epsilon} \\
&+\frac{F\left[f+\epsilon \phi_{1}\right]-F[f]}{\epsilon}
\end{aligned}
$$

the three fractions differ respectively from the quantities $D\left[f, \phi_{1}+\phi_{2}\right], D\left[f+\epsilon \phi_{1}, \phi_{2}\right]$ and $D\left[f, \phi_{1}\right]$ by infinitesimals less in numerical value than $\eta_{\phi_{1}+\phi_{2}}(\epsilon), \eta_{\phi_{2}}(\epsilon)$ and $\eta_{\phi_{1}}(\epsilon)$ respectively. And since $\phi_{1}$ and $\phi_{2}$ are given, these infinitesimals all approach zero with $\epsilon$ by (II). Hence

$$
\begin{aligned}
D\left[f, \phi_{1}+\phi_{2}\right] & =\lim _{\epsilon=0} D\left[f+\epsilon \phi_{1}, \phi_{2}\right]+D\left[f, \phi_{1}\right] \\
& =D\left[f, \phi_{2}\right]+D\left[f, \phi_{1}\right] .
\end{aligned}
$$

This is the distributive property required for the proof of Theorem 1.

3. Proof of Theorem 2. Under the hypotheses of Theorem 2, since (III) implies (I), the first variation takes the form (1) of Theorem 1. We may assume that at interior points of $(a, b)$ the discontinuities of $\alpha_{f}(x)$ are regular, that is, that $\alpha_{f}(x)=\left[\alpha_{f}(x+0)+\alpha_{f}(x-0)\right] / 2$, since the value of $\alpha_{f}(x)$ at an interior point of discontinuity does not affect the value of the integral. Theorem 2 may be proved by means of another theorem.

THEOREM 3. If the first variation of $F[f]$ has the Stieltjes form (1) and (III) is valid when $f_{2}=f$, the first variation may also be written in the form (2).

It will be shown that $\alpha_{f}(x)$, with the possible discontinuities regularized as just indicated, is absolutely continuous. For suppose this function is not absolutely continuous. Then, given $\mu>0$, there is a sequence of finite sets of non-overlapping intervals

$\left\{\left(a_{1}^{(m)}, b_{1}^{(m)}\right),\left(a_{2}^{(m)}, b_{2}^{(m)}\right), \cdots,\left(a_{j_{m}}^{(m)}, b_{j_{m}}^{(m)}\right)\right\}, \quad(m=1,2, \cdots)$,

such that 


$$
\sum_{i=1}^{j_{m}}\left\{\alpha_{f}\left(b_{i}{ }^{(m)}\right)-\alpha_{f}\left(a_{i}{ }^{(m)}\right)\right\} \mid>\mu, \quad(m=1,2, \cdots)
$$

where

$$
\lim _{m=\infty} \sum_{i=1}^{j_{m}}\left(b_{i}^{(m)}-a_{i}^{(m)}\right)=0 .
$$

Let $C$ be a positive constant, and consider the continuous functions $C_{a^{\prime} b^{\prime}, n}$ defined as follows:

(i) If $a<a^{\prime}, b^{\prime}<b, n$ is great enough so that $a+1 / n<a^{\prime}$, $a^{\prime}+2 / n<b^{\prime}, b^{\prime}+1 / n<b$, and

$$
\begin{aligned}
C_{a^{\prime} b^{\prime}, n} & =C, \quad a^{\prime}+\frac{1}{n} \leqq x \leqq b^{\prime}-\frac{1}{n}, \\
& =0, \quad a \leqq x \leqq a^{\prime}-\frac{1}{n}, \quad b^{\prime}+\frac{1}{n} \leqq x \leqq b^{\prime},
\end{aligned}
$$

and the functions $C_{a^{\prime} b^{\prime}, n}$ are linear functions over the intervals $\left(a^{\prime}-1 / n, a^{\prime}+1 / n\right),\left(b^{\prime}-1 / n, b^{\prime}+1 / n\right)$, joining continuously on to the end values already defined.

(ii) If $a=a^{\prime}, b^{\prime}<b, n$ is great enough so that $b^{\prime}+1 / n<b$, $a^{\prime}+1 / n<b^{\prime}$, and

$$
\begin{aligned}
C_{a^{\prime} b^{\prime}, n} & =C, & & a \leqq x \leqq b^{\prime}-\frac{1}{n}, \\
& =0, & & b^{\prime}+\frac{1}{n} \leqq x \leqq b^{\prime}
\end{aligned}
$$

and $C_{a^{\prime} b^{\prime}, n}$ is a linear function over $\left(b^{\prime}-1 / n, b^{\prime}+1 / n\right)$, joining continuously on to the end values already defined.

(iii) If $a<a^{\prime}, b^{\prime}=b$ or if $a^{\prime}=a, b^{\prime}=b$, the definition is again modified in a manner analogous to (ii).

In the case (i) we have

$$
\begin{aligned}
& \lim _{n=\infty} \int_{a}^{b} C_{a^{\prime} b^{\prime}, n} d \alpha(x) \\
& \quad=C\left[\frac{\alpha\left(b^{\prime}+0\right)+\alpha\left(b^{\prime}-0\right)}{2}-\frac{\alpha\left(a^{\prime}+0\right)+\alpha\left(a^{\prime}-0\right)}{2}\right] \\
& \quad=C\left[\alpha\left(b^{\prime}\right)-\alpha\left(a^{\prime}\right)\right],
\end{aligned}
$$


and the same final expression remains valid in the cases (ii) or (iii). Hence for a given $m$ the quantity $T$,

$T=\lim _{n=\infty} D\left[f, \quad \sum_{i=1}^{j_{m}} C_{a_{i}{ }^{(m)} b_{i}{ }^{(m)}, n}\right]=\lim _{n=\infty} \sum_{i=1}^{j_{m}} \int_{a}^{b} C_{a_{i}{ }^{(m)} b_{i}{ }^{(m)}, n} d \alpha_{f}(x)$,

has the value

$$
T=C \sum_{i=1}^{j_{m}}\left(\alpha\left(b_{i}{ }^{(m)}\right)-\alpha\left(a_{i}^{(m)}\right)\right)>C \mu .
$$

But also, for a given $m$, if we write

$$
\begin{gathered}
\Delta F=F\left[f+\epsilon \sum_{i=1}^{j_{m}} C_{a_{i}{ }^{(m)} b_{i}{ }^{(m)}, n}\right]-F[f], \\
I_{n}=\sum_{i=1}^{j_{m}} \int_{a}^{b} C_{a_{i}{ }^{(m)} b_{i}{ }^{(m)}, n} d x
\end{gathered}
$$

we have, by (III), $\Delta F \leqq \epsilon M I_{n}$, or $\Delta F / \epsilon \leqq M I_{n}$, and by letting $\epsilon$ approach zero, the same quantity $D$ just used above is seen to have a value

$$
\lim _{\epsilon=0} \frac{\Delta F}{\epsilon} \leqq M I_{n}
$$

Hence as $n$ tends to infinity

$$
T \leqq M C \sum_{i=1}^{j_{m}}\left(b_{i}{ }^{(m)}-a_{i}^{(m)}\right) .
$$

But for $m$ great enough, the right hand member of (6) can be made as small as we please, by (4); and (5) and (6) are in contradiction. Hence $\alpha_{f}(x)$ must be absolutely continuous. If we define $\beta_{f}(x)$ as the derivative of $\alpha_{f}(x)$ where it exists, and as, say, zero otherwise, we shall have then, as is well known,

$$
\begin{aligned}
\alpha_{f}(x) & =\text { const. }+\int_{a}^{x} \beta_{f}(x) d x, \\
\int_{a}^{b} \phi(x) d \alpha_{f}(x) & =\int_{a}^{b} \phi(x) \beta_{f}(x) d x .
\end{aligned}
$$

The proof of Theorem 3 is thus complete. Theorem 2 is a corollary of Theorem 3. It may be remarked that in these 
theorems $\beta(x)$ is merely summable, and that therefore they are not special cases of theorems where the corresponding function is summable with its square.

4. Another Theorem. In the proof of Theorem 1, it is not sufficient to substitute for (II) the weaker condition by which merely the existence of $D\left[f_{1}, \phi\right], f_{1}$ in $R$, is demanded, since the situation must contain as a particular case that of the function $F\left(y_{1}, y_{2}\right)$ of two independent variables, in the usual sense. But instead of the requirement of uniformity in (II) may be substituted other simple conditions. Thus we have the theorem:

THEOREM 4. If $F\left[f_{1}\right]$ is continuous for $f_{1}$ in the neighborhood of the particular function $f$, if $D\left[f_{1}, \phi\right]$ exists for $f_{1}$ in the neighborhood of $f$, with $\phi$ arbitrary, and if $D\left[f_{1}, \phi\right]$ is continuous in $f_{1}$ at $f_{1}=f$, with $\phi$ arbitrary but fixed, then the formula (1) is valid; and if also

$$
\left|F\left[f_{1}\right]-F[f]\right| \leqq M \int_{a}^{b}\left|f_{1}-f\right| d x,
$$

the formula (2) is valid.

In fact, since $F\left[f_{1}\right]$ is continuous in particular at $f_{1}=f$, we have, by definition, an $M$ such that

$$
\left|F\left[f_{1}\right]-F[f]\right| \leqq M \max \left|f_{1}-f\right|,
$$

whence $D[f, \phi] \leqq M \max |\phi|$.

Also, the functions $F\left[f+\epsilon\left(\phi_{1}+\phi_{2}\right)\right], \quad F\left[f+\epsilon_{1} \phi_{1}+\epsilon \phi_{2}\right]$, $F\left[f+\epsilon \phi_{1}\right]$ are all continuous functions of $\epsilon$ and differentiable with respect to $\epsilon$. In particular, for $0<\omega^{\prime}<\epsilon$,

$$
F\left[f+\epsilon_{1} \phi_{1}+\epsilon \phi_{2}\right]-F\left[f+\epsilon_{1} \phi_{1}\right]=\epsilon D\left[f+\epsilon_{1} \phi_{1}+\omega^{\prime} \phi_{2}, \phi_{2}\right],
$$

an equation in which we may write $\epsilon_{1}=\epsilon$. Consequently, the law of the mean applied to (3) yields the equation

$D\left[f+\omega\left(\phi_{1}+\phi_{2}\right), \phi_{1}+\phi_{2}\right]=D\left[f+\epsilon \phi_{1}+\omega^{\prime} \phi_{2}, \phi_{2}\right]+D\left[f+\omega^{\prime \prime} \phi_{1}, \phi_{1}\right]$

where $\omega, \omega^{\prime}, \omega^{\prime \prime}$ are contained between 0 and $\epsilon$. And since $D\left[f_{1}, \phi\right]$ is continuous at $f_{1}=f$, we can let $\epsilon$ approach zero, and obtain the relation $D\left[f, \phi_{1}+\phi_{2}\right]=D\left[f, \phi_{2}\right]+D\left[f, \phi_{1}\right]$. But this is the distributive property required in the proof of Theorem 1 , and the rest of the analysis proceeds as before. 
5. Conclusion. It is worthy of remark that by means of the property (III), alone, the field of functions to which $F[f]$ is applicable may be considerably extended. If (III) is valid, $F[f]$ may be extended to all functions $f$ in $R$ summable Lebesgue. And if $\Phi_{1}(x) \equiv-\infty, \quad \Phi_{2}(x) \equiv+\infty$, the functional may be extended to all functions summable Lebesgue.

In fact, under (III), the functional has the same fundamental property as integration with respect to sequences $f_{n}(x)$ with limit $f(x)$; if the absolute continuity of $\int f_{n}(x) d x$ is uniform in $n$ and $\lim f_{n}(x)=f(x)$, it follows that $\lim F\left[f_{n}\right]$ exists and is independent of the choice of the sequence $f_{n}$. Hence $F[f]$ may be defined as the limit of $F\left[f_{n}\right]$. And thus in successive steps, and by writing $F\left[f_{1}\right]=F\left[f_{2}\right]$, if $f_{1}$ and $f_{2}$ differ only on a set of zero measure, the extension is completed.

The Rice Institute

\title{
THE EVALUATION OF CERTAIN DEFINITE INTEGRALS BY THE USE OF PROBABILITY FUNCTIONS*
}

\author{
BY W. D. BATEN
}

1. Introduction. The object of this paper is to present three methods of evaluating certain definite integrals by using probability functions. The first method consists in finding the probability law or function for the sum of $n$ independent variables, which are each subject to given probability laws, by Mayr's method $\dagger$ and comparing this with the probability for the same sum obtained by Dodd's method. On equating the two results thus obtained one often finds the value of certain integrals.

Method II consists in finding the probability law for $n$ independent variables, which is expressed as an integral, and then allowing $n$ to be equal to 1 .

\footnotetext{
* Presented to the Society, August 29, 1929.

$\dagger$ Karl Mayr, Wahrscheinlichkeitsfunktionen und ihre Anwendungen, Monatshefte für Mathematik und Physik, vol. 30.

$\ddagger \mathrm{E}$. L. Dodd, The frequency law of a function of one variable, this Bulletin, vol. 31 (1925), pp. 27-31; The frequency law of a function of variables with given frequency laws, Annals of Mathematics, (2), vol. 27, pp. 12-20.
} 